\title{
The deconfinement phase transition, hadronization and the NJL model
}

\author{
Sibaji Raha* \\ * Physics Department, Bose Institute \\ 93/1, Acharya Prafulla Chandra Road \\ Calcutta 700 009, INDIA \\ Electronic Mail : sibaji@bosemain.boseinst.ernet.in
}

\begin{abstract}
One of the confident predictions of QCD is that at sufficiently high temperature and/or density, hadronic matter should undergo a thermodynamic phase transition to a colour deconfined state of matter - popularly called the Quark-Gluon Plasma (QGP). In low energy effective theories of Quantum Chromodynamics (QCD), one usually talks of the chiral transition for which a well defined order parameter exists. We investigate the dissociation of pions and kaons in a medium of hot quark matter decsribed by the Nambu - Jona Lasinio (NJL) model. The decay widths of pion and kaon are found to be large but finite at temperature much higher than the critical temperature for the chiral (or deconfinement) transition, the kaon decay width being much larger. Thus pions and even kaons (with a lower density compared to pions) may coexist with quarks and gluons at such high temperatures. On the basis of such premises, we investigate the process of hadronization in quarkgluon plasma with special emphasis on whether such processes shed any light on acceptable low energy effective theories of QCD.
\end{abstract}

Quantum Chromodynamics (QCD) is believed to be the underlying theory of strong interactions. Although enormously successful, inasmuch as it is part of the standard model of physical interactions, the applicability of QCD is studying nuclear interactions or hadronic processes at low energies is still limited. The difficulties are largely technical, associated with the non-perturbative features of the theory and hence, a large amount of effort is being devoted to finding effective lagrangians which contain features compatible with the low energy limit of QCD. This is the thrust of the present workshop. The philosophy of my talk is somewhat complementary; I am going to discuss QCD phenomena which occur in very energetic nuclear collisions, where one hopes perturbative QCD does play an important role, at least during the initial time period.

A confident prediction of QCD is that at very high temperature and/or density, the bulk properties of strongly interacting matter would be governed by the coloured QCD degrees of freedom - quarks and gluons- rather than the usual hadrons. Such a phase is called quark gluon plasma (QGP) [1] in the literature. Conditions conducive to the formation of QGP may have existed in the early universe during the first few microseconds after the Big Bang. Also, such conditions may be transiently created in highly energetic collsions of large nuclei and the search for such a novel phase of matter constitutes a major area of current research in the field of high energy physics.

Whether QGP is separated from the hadronic world by an actual thermodynamic phase transition is an open question. It has been widely postulated that such a phase transition may indeed occur, where the quarks and gluons convert into colourless hadrons. Recent results, showing the lack of thermodynamic equilibrium [2] in the quark-gluon phase in ultrareletivistic heavy ion collisions, indicate however that such an ideal situation is unlikely. It should also be noted that although the persistence of non-perturbative effects till very high temperatures was suggested in the literature quite early on [3], it is only recently that the lattice results have confirmed that non-perturbative hadron like excitations could survive at temperatures far above the chiral phase transition temperature [i for pion screening mass has been studied in ref. [5]. The analysis of [5] has been contradicted by Boyd et al. in ref. [6]. The conclusion of these authors [6] is consistent with the existence of free quarks at high temperatures. On the other hand, Shuryak [7] argued in a subsequent work that the non-perturbative modes, especially pion- like excitations, could indeed survive till temperatures above $T_{c}$. Furthermore, similar results for pion screening masses are obtained in $\sigma$ - model as well [6]. It is thus imperative to understand the behaviour of such hadronic resonances, their formation, stability and so on, in a quark gluon medium at high temperature. We confine our attention to the case of pions and kaons only; these, being lighter than other hadrons, account for the bulk of the multiplicity.

Formation of light mesons like pions and kaons, a bound state of light relativistic quarks, is an extremely difficult problem to handle in QCD, where all the troublesome features of non-perturbative QCD appear. We therefore employ the usual practice of looking at the pion and kaon as Goldstone bosons arising from the spontaneous breaking of the chiral symmetry, a feature most suitably addressed in the Nambu Jona-Lasinio (NJL) model [8].

The formulation of NJL model in flavour SU(3) was first introduced by Hatsuda et al. [9] and Bernard et al. [10]. The three flavour NJL model Lagrangian is written in terms of $u, d$ and $s$ quarks, the interaction between them being constrained by the $S U(3)_{L} \otimes S U(3)_{R}$ chiral symmetry, explicit symmetry breaking due to the current quark masses and the $U(1)_{A}$ breaking due to the axial anamoly [9]. The full Lagrangian with KMT (Kobayashi- Maskawa -'t-Hooft) term is given below [9]. 


$$
\begin{aligned}
\mathcal{L} & =\bar{q}(i \gamma \cdot \partial-\mathbf{m}) q+\frac{1}{2} g_{s} \sum_{a=0}^{8}\left[\left(\bar{q} \lambda_{a} q\right)^{2}+\left(\bar{q} i \lambda_{a} \gamma_{5} q\right)^{2}\right] \\
& +g_{D}\left[\operatorname{det} \bar{q}_{i}\left(1-\gamma_{5}\right) q_{j}+\text { h.c. }\right]
\end{aligned}
$$

where the quark fields $q_{i}$ has three colours $\left(N_{c}=3\right)$ and three flavours $\left(N_{f}=3\right)$ and $\lambda_{a}(a=1,8)$ are the Gell-Mann matrices. The quark mass matrix is given by $\mathbf{m}=\operatorname{diag}\left(m_{u}, m_{d}, m_{s}\right)$.

In the mean field approximation, the quark condensates at finite temperature are given by,

$$
<<\bar{q}_{i} q_{i}>>=-2 N_{c} \int \frac{d^{3} p}{2 \pi^{3}} \frac{M_{i}}{E_{i p}} f\left(E_{i p}\right)
$$

where $E_{i p}$ is the quark single particle energy for the i-th specie and $f\left(E_{i p}\right)=1-n_{i p}-\bar{n}_{i p}, n_{i p}$ and $\bar{n}_{i p}$ being the Fermi-Dirac distributions for quarks and anti-quarks, respectively. If quark chemical potential is zero, then $n_{i p}=\bar{n}_{i p}=\left[\exp \left(E_{i p} / T\right)+1\right]^{-1}$.

The temperature dependent constituent quark masses $M_{i}$ are obtained from the expressions below,

$$
\begin{gathered}
M_{u}=m_{u}-2 g_{s} \alpha-2 g_{D} \beta \gamma \\
M_{d}=m_{d}-2 g_{s} \beta-2 g_{D} \alpha \gamma \\
M_{s}=m_{s}-2 g_{s} \gamma-2 g_{D} \alpha \beta
\end{gathered}
$$

where

$$
<<\bar{u} u>>\equiv \alpha, \quad<<\bar{d} d>>\equiv \beta, \quad<<\bar{s} s>>\equiv \gamma
$$

We now want to investigate the decay of pionic and kaonic excitations, the properties of which we assume to be given by the NJL model. It should be mentioned here that at temperatures above the critical temperature, these mesonic excitations are more like resonances with large effective masses [4. 5]. In the following, we study [11] the decay width of such pseudoscalar excitations in the hot quark medium as a function of temperature, starting with the Lagrangian given above in equation (11).

The quark mass $M_{i}$ appearing in eq. (2) and in eq. (3) is a very important ingredient in our calculation. In the absence of any medium and/or dynamic effect, $M_{i}$ is the current quark mass. On the other hand, we know that due to the spontaneous breakdown of the chiral symmetry, quarks attain the value of the constituent quark mass [12].

In the present calculation we have used the parametrisation of ref. [10] $\left(\Lambda=631.4, g_{s} \Lambda^{2}=3.67, g_{D} \Lambda^{5}=-9.29\right.$ and current mass $m_{u, d}\left(m_{s}\right)=5.5(135.7) \mathrm{MeV}$ ) to calculate the quark and meson masses. The constituent quark masses are calculated using the gap equations(eq. 3). These quark masses are then put into the dispersion equation for mesons to get dynamical masses of mesons ( $\pi$ and $K$, here).

$$
1+2 G_{\phi} \Pi_{i j}(\omega, \vec{q} \rightarrow 0)=0
$$

where $\Pi_{i j}$ is the one loop polarization due to $u$ and $d$ quark for pions and $u$ or $d$ and $s$ quark for kaons. $G_{\phi}$ is the coupling constant with $\phi$ coerresponding to $\pi$ or $K$. The general expression for polarization function is

$$
\begin{array}{r}
\Pi\left(q_{0}, \vec{q}\right)=\frac{N_{c}}{(2 \pi)^{3}} \int_{0}^{\Lambda} \frac{d^{3} p}{E_{p} E_{k}}\left[\left(n_{k}-n_{p}\right)\left\{\frac{1}{E_{p}-E_{k}+q_{0}+i \epsilon}+\frac{1}{E_{p}-E_{k}-q_{0}-i \epsilon}\right\}\right. \\
\times\left(-E_{p} E_{k}+\vec{p} \cdot \vec{k}+M_{1} M_{2}\right) \\
+\left(n_{k}+n_{p}-1\right)\left\{\frac{1}{E_{p}+E_{k}+q_{0}+i \epsilon}+\frac{1}{E_{p}+E_{k}-q_{0}-i \epsilon}\right\} \\
\left.\times\left(E_{p} E_{k}+\vec{p} . \vec{k}+M_{1} M_{2}\right)\right]
\end{array}
$$

where $N_{c}$ is the number of colours and $\vec{k}=\vec{p}+\vec{q}$. The energies $E_{p}=\sqrt{p^{2}+M_{1}{ }^{2}}$ and $E_{k}=\sqrt{(\vec{p}+\vec{q})^{2}+M_{2}{ }^{2}}$. For pion, $M_{1}=M_{2}=M_{u}$. For kaon, $M_{1}=M_{u(d)}$ and $M_{2}=M_{s} . n_{k}$ and $n_{p}$ are the Fermi-Dirac distribution functions defined earlier. The pseudoscalar couplings are,

$$
\begin{aligned}
& G_{\pi}=g_{s}+g_{D} \gamma \\
& G_{K^{ \pm}}=g_{s}+g_{D} \beta \\
& G_{K^{0}}=g_{s}+g_{D} \alpha
\end{aligned}
$$


where $\alpha, \beta$ and $\gamma$ are defined in eq. (4).

The decay width is evaluated using the imaginary part of the eq.(6) as given below,

$$
\Gamma_{\phi}=-\frac{G_{\phi q}{ }^{2} \operatorname{Im} \Pi(\omega, \vec{q} \rightarrow 0)}{\omega}
$$

where $G_{\phi q}$ is the empirical meson-quark coupling as obtained in NJL. Here we have used $G_{\pi q}=3.5$ and $G_{K q}=3.6$ [10].
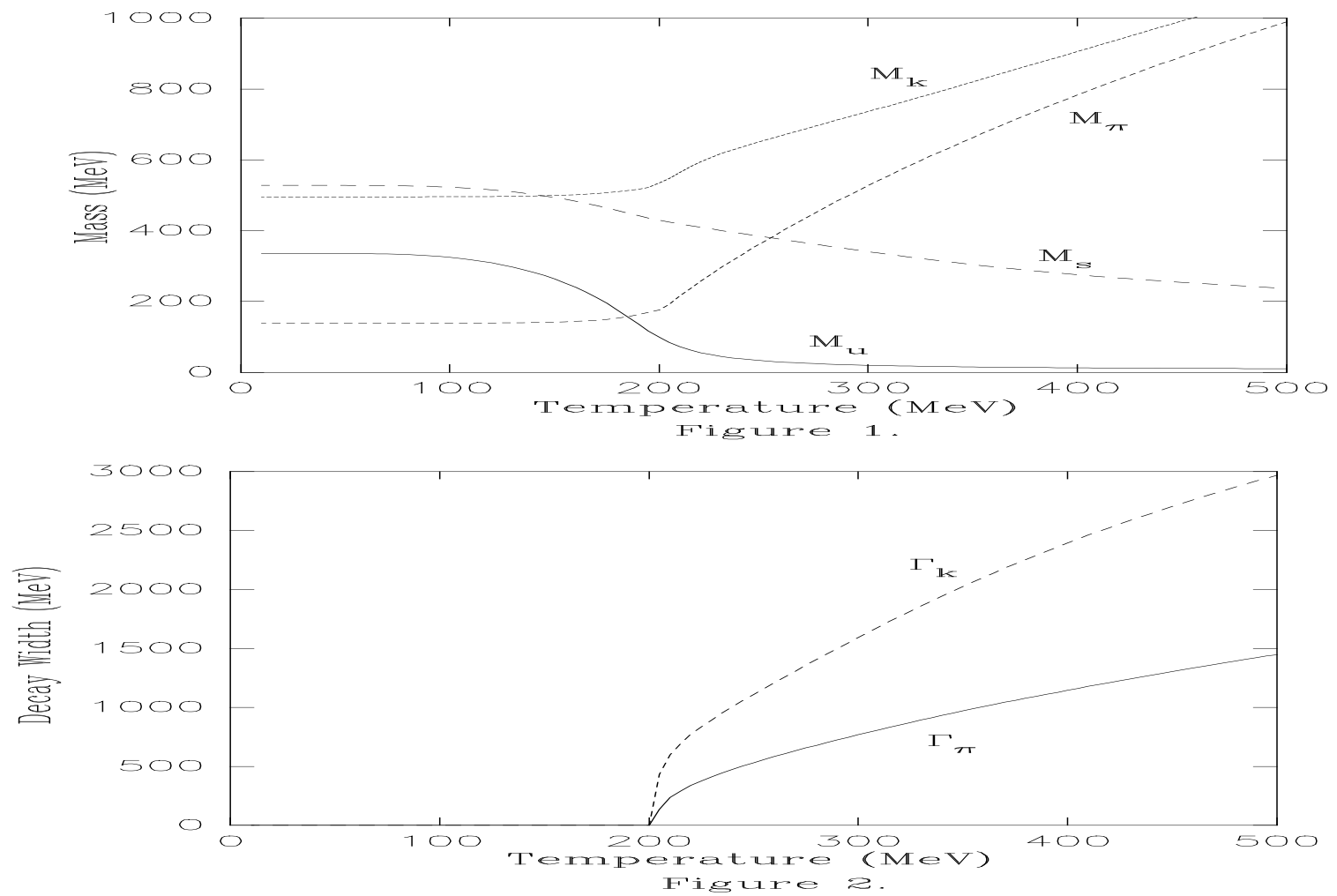

Figure 1: Variation of quark and meson masses with temperature.

Figure 2: Variation of meson decay widths with temperature.

The variation of quark and meson masses is shown in figure 1. The $u$ or $d$ quark masses starting from $135 \mathrm{MeV}$ drops to the current quark mass value just after a temperature of $200 \mathrm{MeV}$. On the other hand, the drop in the strange quark mass is much smaller around that temperature, showing the effect of explicitly broken chiral symmetry, by a larger amount, in the SU(3) sector. Pion and kaon both show a similar qualitative behaviour. The masses of pion and kaon remain constant at their free masses upto a temperature of $200 \mathrm{MeV}$ but increases sharply after that, the pion mass rising to $900 \mathrm{MeV}$ and kaon mass to $1000 \mathrm{MeV}$ around a temperature of $450 \mathrm{MeV}$, thus giving a slower increment for kaons compared to pions. The difference in the behaviour of kaon and pion can be attributed to the difference in $u$ and $s$ quark masses.

Figure 2 shows that the decay width, for both pion and kaon, is very high at high temperature and decreases with decreasing temperature, going to zero at around $T=0.2 \mathrm{GeV}$. It is worth noticing that at around the same temperature, the effective pion mass attains the value of the free pion mass (figure 1). The decay width of kaon is around $3 \mathrm{GeV}$ where as that of pion is around $1.4 \mathrm{GeV}$ at $\mathrm{T}=500 \mathrm{MeV}$. This is very significant for two reasons. Firstly, our results show that though there will be pions and kaons along with the quarks at high temperature phase, the numbers of mesons will be very small due to their large decay width. Moreover, the number of kaons will be much less compared to pions at high temperature phase, though both the mesons will become stable around the same temperature (below $200 \mathrm{MeV}$ ).

We thus find that even without any consideration of the detailed evolution and dynamics of the system, the mesonic modes in a hot quark medium are found to become important around a temperature of $200 \mathrm{MeV}$. Though the question whether this is a signature of a phase transition cannot be addressed within the present framework, the fact that most 
of the pions and kaons decay into quarks, owing to a large decay width at temperatures higher than $T=200 \mathrm{MeV}$, is a remarkable finding. Moreover, both the pionic as well as kaonic modes start becoming important at about the same temperature, thus providing a hint of some kind of a transition temperature. It is therefore tempting to attempt a microscopic investigation of the process of hadronization within the NJL model.

Recently there have been some attempts to study the formation of hadrons in quark matter using different semimicroscopic approaches. These studies can be characterized either as model dependent calculations [13], or the computer codes based on the string phenomenology [14], or other phenomenological description of hadronization [15]. None of these approaches account for the essential lack of equilibrium in the quark-gluon phase. Some efforts have also been made to estimate hadronization within the parton cascade model by introducing a cut-off to mimic the non-perturbative effects [16]. To the best of our knowledge, the first study aimed at investigating the dynamical process of hadronization in a non-equilibrated quark-gluon system from a physically transparent approach was in [17].

We have earlier shown [2] that perturbative estimates of the gluon-gluon, quark-gluon and quark-quark cross sections allow us to study the evolution of the quark-gluon matter formed in ultrarelativistic heavy ion collisions by visualizing the quarks as Brownian particles in a hot gluonic thermal bath. Let us start with such a premise, which, in our opinion, describes the non-equilibrium aspects of the evolution in a physically transparent manner.

Hadronization in such a system can be studied in the light of Smoluchowski's theory of coagulation in colloids, which was elaborated further by Chandrasekhar [18]. This theory suggests that coagulation results as a consequence of each colloidal particle being surrounded by a sphere of influence of a certain radius $R$ such that the Brownian motion of a particle proceeds unaffected only so long as no other particle comes within its sphere of influence. When another Brownian particle does come within a distance $R$ of the test particle, they form a two body cluster. This cluster also describes a Brownian motion but at a reduced rate due to its increased size/mass. The process continues further till a single cluster of all the particles is formed.

In order for this stochastic scenario of cluster formation to apply to a system of Brownian quarks in the hot gluon bath, it is essential that each quark has an appropriate sphere of influence of radius $r$. Obviously, this radius $r$ will depend on the spin-isospin combination of final cluster (whether the final cluster is scalar, pseudoscalar, vector or axial vector meson or even a baryon). Mesons are formed when one quark and one antiquark with proper quantum numbers come within the spheres of influence of each other. (Clusters with greater numbers of quarks and antiquarks (e.g. baryons) can also be formed by imposing the conditions of colour neutrality and charge balance properly.) This implies that the radius of the sphere of influence corresponds to the correlation length between the quarks in the proper hadronic channel. In other words, this is the screening length of the corresponding hadrons in the hot quark-gluon matter. (One can immediately see, without further ado, that the rate of pion formation in the hot quark matter should be rather small at high temperatures and increase with falling temperature as the pion screening length (inverse of the screening mass) decreases with increasing temperature in all realistic pictures.) The pions formed at very high temperatures are most likely to decay back into quarks and antiquarks, because of the large decay width at high temperatures. We adopt, for the sake of consistency, the NJL model estimates for the pion screening mass [19] in the present work.

The rate of pion formation from Brownian quarks as a stochastic process, as is evident from the preceding discussion, depends on the number of quarks (antiquarks) falling into the sphere of influence of another antiquark (quark), the number of pions decaying back to quarks and antiquarks and also the change in the pion density due to the expansion of the system. Thus the rate equations can be written as,

$$
\begin{array}{r}
\frac{d n_{\pi^{a}}}{d t}=n_{q_{i}} n_{\bar{q}_{j}} 4 \pi r^{2}<\vec{v} \cdot \hat{r}>-\Gamma_{\pi^{a} \rightarrow q_{i} \bar{q}_{j}}^{\text {total }}-\frac{n_{\pi^{a}}}{t} \\
\frac{d n_{\pi^{0}}}{d t}=\frac{1}{2}\left(n_{u} n_{\bar{u}}+n_{d} n_{\bar{d}}\right) 4 \pi r^{2}<\vec{v} \cdot \hat{r}>-\Gamma_{\pi^{0} \rightarrow u \bar{u}(d \bar{d})}^{\text {total }}-\frac{n_{\pi^{0}}}{t} \\
\frac{d n_{q_{i}}}{d t}=\Gamma_{g \rightarrow q_{i} \bar{q}_{i}}^{\text {total }}+\Gamma^{\text {total }}{ }_{g g \rightarrow q_{i} \bar{q}_{i}}-n_{q_{i}} n_{\bar{q}_{j}} 4 \pi r^{2}<\vec{v} \cdot \hat{r}>+\Gamma_{\pi \rightarrow q_{i} \bar{q}_{j}}^{t o t a l}-\frac{n_{q_{i}}}{t}
\end{array}
$$

In eqs. (99 10), the first term is the rate of pion formation $(a \equiv+$ or -$)$; the second term is the rate of pions decaying back to quarks and the third term is due to Bjorken (longitudinal) expansion of the system. $i(j)$ stands for $u$ or $d$ (we ignore $s$ and other heavier flavours). $\langle\vec{v} \cdot \hat{r}\rangle$ (the average relative velocity in the radial direction) is calculated using the Jüttner distribution,

$$
f(x, p)=e^{-\beta p \cdot u(x)}
$$

There would also be a corresponding rate equation for the antiquarks, which looks exactly like eq. (11) and hence not explicitly written. In eq. (11) the $\Gamma^{\text {total }}{ }_{g \rightarrow q \bar{q}}$ as well as $\Gamma^{\text {total }}{ }_{g g \rightarrow q \bar{q}}$ stand for the corresponding net quantities.

As mentioned earlier, we are considering a non-equilibrated quark matter and hence the pions formed will also be out of equilibrium. This is taken into account by multiplying the relevant distribution functions with the ratios 
$r_{q}=n_{q} / n_{e q}$ and $r_{\pi}=n_{\pi} / n_{e \pi}$ where $n_{q}$ and $n_{e q}$ are non-equilibrium and equilibrium densities of quarks and $n_{\pi}$ and $n_{e \pi}$ are non-equilibrium and equilibrium densities for pions.

In all these expressions, the appropriate masses are the effective masses including the current as well as thermal contribution, , whose importance in determining the dynamics of the hot quark matter has been well established. For quarks (antiquarks), this is

$$
m_{\text {eff }}=\sqrt{m_{q}(\text { curr })^{2}+m_{q}(\text { thermal })^{2}}
$$

where [20,21]

$$
m_{q}^{2}(\text { thermal })=\left(1+\frac{r_{q}}{2}\right)\left(\frac{g_{s} T}{3}\right)^{2}
$$

and $m_{q}($ curr $)$ is taken to be $10 \mathrm{MeV}$. For gluons the thermal mass is,

$$
m_{g}(\text { thermal })=\frac{2}{3} g_{s} T
$$

The running coupling constant $\alpha_{s}$ as a function of temperature is given by [22]

$$
\alpha_{s}=\frac{12 \pi}{\left(33-2 n_{f}\right) \ln \left[\frac{\bar{Q}^{2}}{\Lambda^{2}}\right]}
$$

with $\bar{Q}^{2}=m_{e f f}{ }^{2}(T)+9 T^{2}$.

Simultaneously, we must take account of energy momentum conservation which, for a Bjorken flow, corresponds to the following equation

$$
\frac{\partial \epsilon}{\partial t}=-\frac{\epsilon+P}{t}
$$

where $\epsilon \equiv \epsilon_{\text {total }}=\epsilon_{g}+\epsilon_{q}+\epsilon_{\pi}$. We also include the one loop correction to $\epsilon_{g}$ [23]. $\epsilon$ and $P$ are related through the velocity of sound, as in [2]. For a complete description of the system, eqs. (9), (10), (11) and (16) must be solved self-consistently. The initial conditions are taken from [2] for RHIC energies. The initial time $\left(t_{g}\right)$ is the time when gluons thermalise $(=0.3 \mathrm{fm})$, where $r_{q}=0.15, r_{g}=1$ and $r_{\pi}$ is taken to be 0 . The temperature at this time is 500 $\mathrm{MeV}$. Note that we are working at $y=0$ so that $t$ and $\tau$ are the same and the baryon chemical potential is zero.
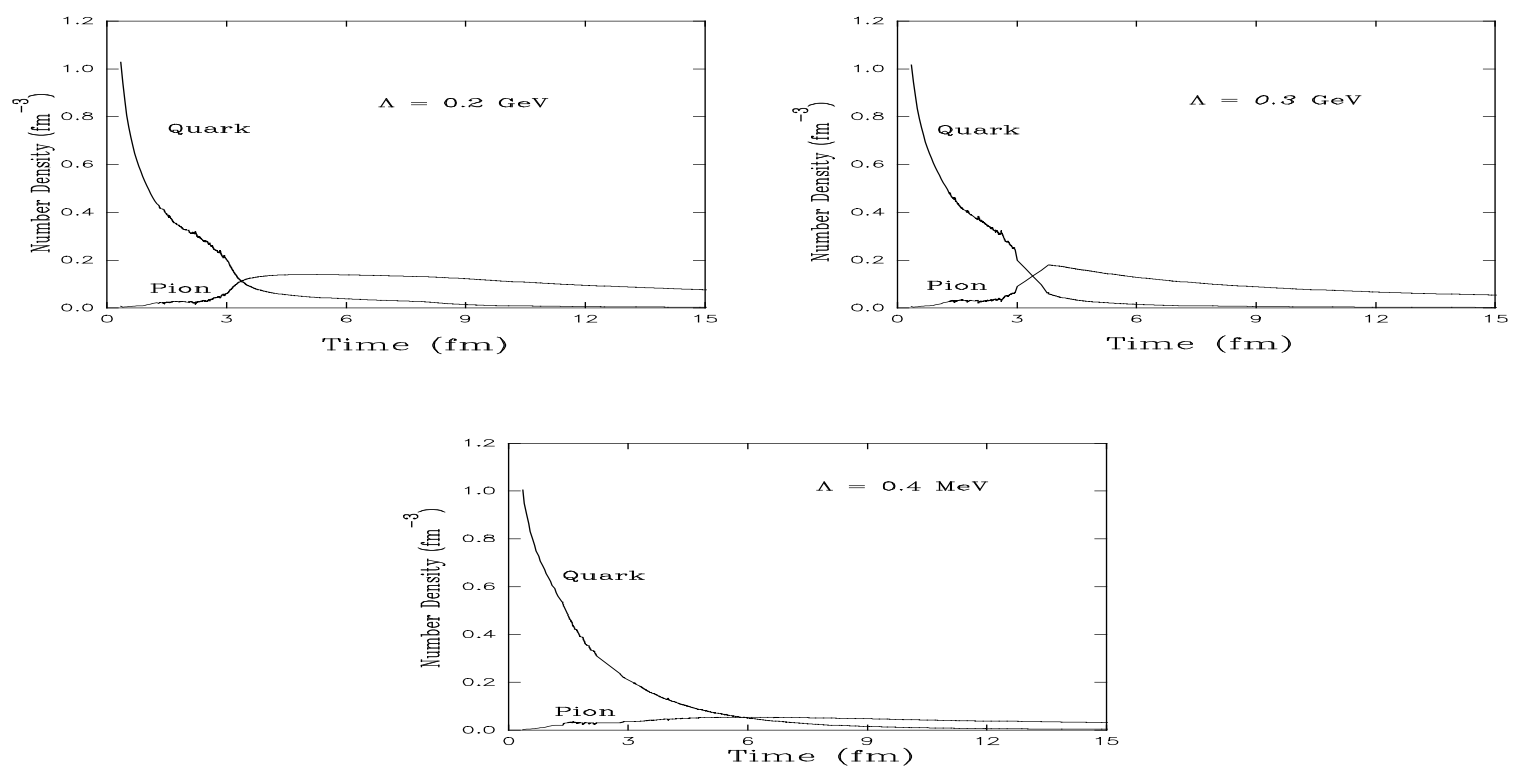

Figure 3 : Time evolution of quark and pion number densities for various values of QCD parameter $\Lambda$. 
Figure 3 shows the variation of pion and quark number densities with time, for various values of the QCD parameter $\Lambda$. In all three cases, we find the same qualitative feature that pions start appearing in the system quite early on but they become appreciable in number only after some time. At late times the system is dominated by pions. This cross over occurs at $t \geq 4 \mathrm{fm}$ for $\Lambda=0.2$ or $0.3 \mathrm{GeV}$ while for $\Lambda=0.4 \mathrm{GeV}$ this happens at $t \sim 6 \mathrm{fm}$.

Figure 4 shows the variation of temperature with time. Obviously, there is a dramatic effect of the QCD parameter $\Lambda$. In all the cases, there is a change at $T \sim 215 \mathrm{MeV}$, corresponding to $t \sim 3.5 \mathrm{fm}$; the variation of temperature with time becomes slower, as is expected in the mixed phase of a first order phase transition. At $\Lambda=0.2 \mathrm{GeV}$, this occurs for a very short period of time, before the system starts cooling again. The duration of the constant temperature configuration increases with $\Lambda$, and for $\Lambda=0.4 \mathrm{GeV}$, it persist upto $9 \mathrm{fm}$ before the temperature of the system starts falling again.

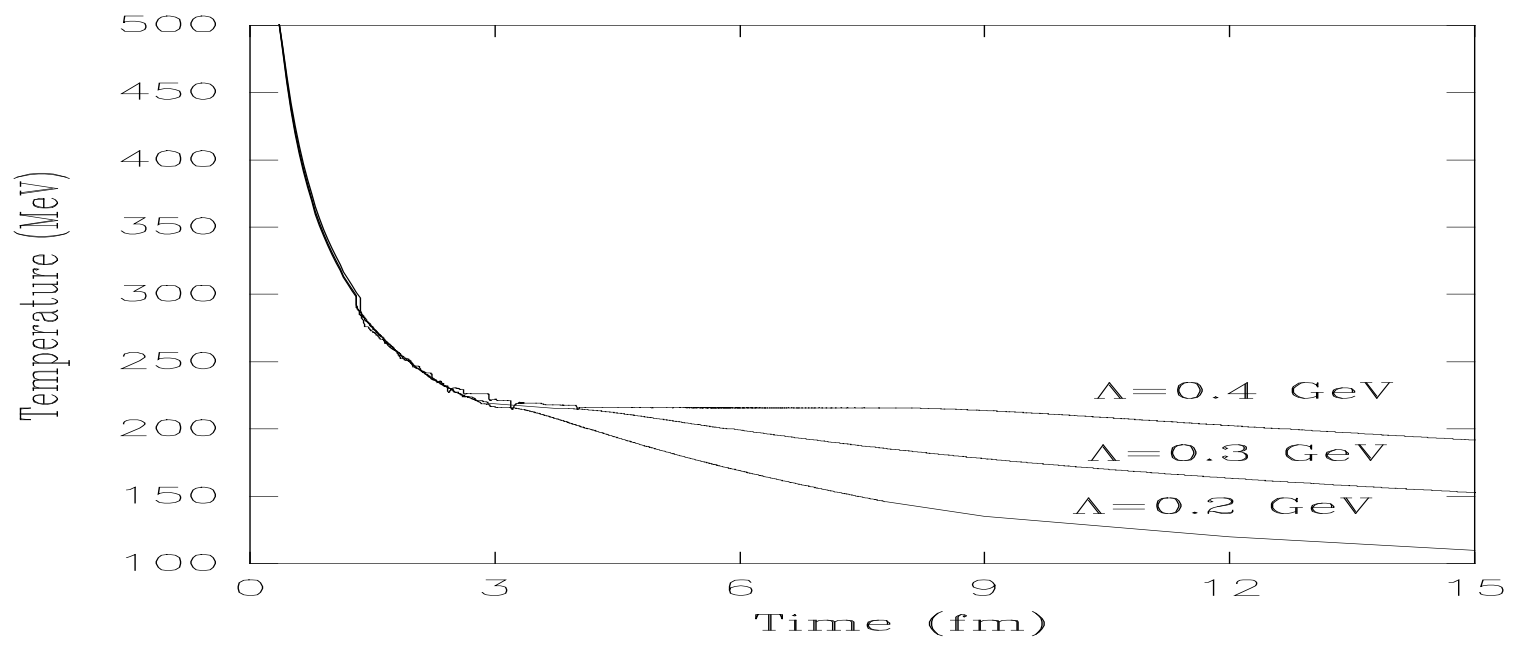

Figure 4: Time evolution of the Temperature for various values of QCD parameter $\Lambda$.

Obviously, this is a clear indication of an apparent first order transition. Microscopically, the appearance of the mixed phase at a temperature of $\sim 215 \mathrm{MeV}$ can be understood from the fact that the pion decay width goes to zero at such a temperature. All the pions that were formed earlier in the system tended to decay back to quarks and antiquarks on a fast time scale. Only after the pion decay width becomes small would the formed pions become stable.

The apparently desirable features of the dynamical deconfining transition seem to arise quite naturally in this scenario. It is therefore important to test if all the conservation laws are obeyed during the evolution. To this end, let us check if entropy increases steadily during the entire evolution.

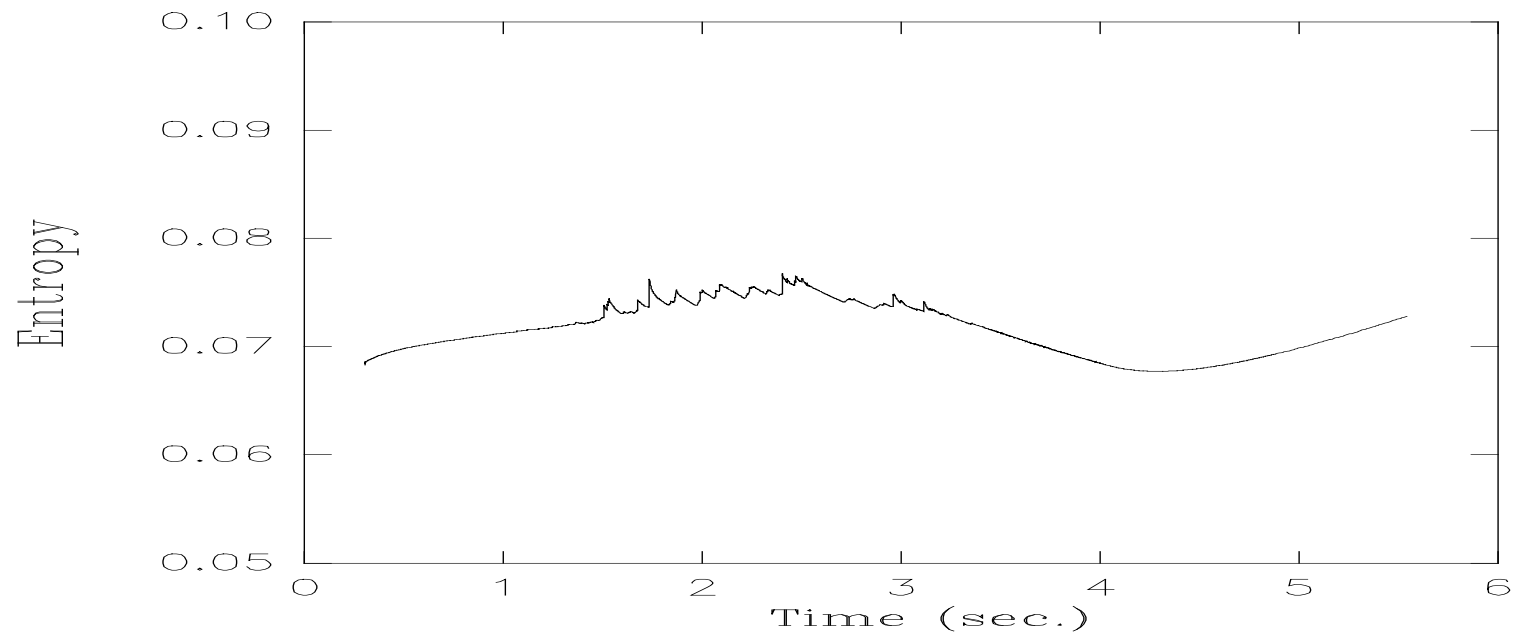

Figure 5 : Time evolution of entropy with time $(\Lambda=0.3 \mathrm{GeV})$. 
The decrease in entropy occurs precisely where the gluons drop out of the system. This, in hindsight, was to be expected, since the sudden decrease in the number of degrees of freedom in the system should lead to a decrease in entropy. The NJL model, in the present form, does not account for the gluonic degrees of freedom. It thus appears to us that in order to obtain an effective low energy lagrangian from QCD capable of describing the dynamical evolution of the quark-gluon system, the role of the gluons must be taken into account. Incorporation of the dilaton field to account for the QCD trace anomaly in the NJL model [24] is indeed a promising step in this respect, but the entropy carried by the gluons at high temperature should also be accommodated. Such a study is on our current agenda.

It is a great pleasure to thank the organisers of the workshop, and especially Prof. J. da Providencia, for the kind invitation and their warm hospitality. This talk is based on a continuing collaboration the author has had with Jan-e Alam, Abhijit Bhattacharyya, Sanjay Kumar Ghosh and Bikash Sinha over the past several years. I take this opportunity to thank them all.

[1] Shuryak, E. V., Phys. Rep. 61, 71 (1980).

[2] Shuryak, E.V., Phys. Rev. Lett. 68, 3270 (1992); Chakraborty, S., Raha, S. and Sinha, B., Mod. Phys. Lett. A7, 927 (1992); Alam, J., Raha, S. and Sinha, B., Phys. Rev. Lett. 73, 1895 (1994); Biro, T.S., Müller, B., Thoma, M. H. and Wang, X.N., Nucl. Phys. A566, 543c (1994); Alam, J. Roy, P., Sarkar, S., Raha, S. and Sinha, B., Int. J. Mod. Phys. A12, 5151 (1997).

[3] Plümer, M., Raha, S., and Weiner, R.M., Phys. Lett. B139, 198 (1984); Nucl. Phys. A418, 549c (1984).

[4] Born, K. et al., Phys. Rev. Lett 67, 302 (1991).

[5] A. Gocksh, Phys. Rev. Lett. 67, 1701 (1991).

[6] Boyd, G., Gupta, S., Karsch, F. and Laermann, E., Z. Phys. C64, 331 (1994).

[7] Shuryak, E.V. and Schaefer, T., Nucl. Phys. B53, 472 (1997).

[8] Nambu, Y and Jona-Lasinio, G., Phys. Rev. 122, 345 (1961).

[9] Kunihiro, T. and Hatsuda, T., Phys. Lett B206, 385 (1988); Phys. Rep. 247, 221 (1994).

[10] Bernard, V., Jaffe, R. L. and Meissner, U - G., Nucl. Phys. B308, 753 (1988).

[11] Bhattacharyya, A., Ghosh, S.K. and Raha, S., Mod. Phys. Lett. A14, 621 (1999).

[12] Frederico, T. and Miller, G. A., Phys. Rev. D45, 4207 (1992).

[13] Rehberg, P., Klevansky, S. P. and Hüfner, J., Phys. Rev. C53, 410 (1996).

[14] Werner, K., Phys. Rep. 232, 87 (1993).

[15] Barz, H.W., Friman, B. L., Knoll, J. and Schulz, H., Nucl. Phys. A519, 831 (1990); Biró, T. S., Lévai, P. and Zimányi, J., Phys. Lett. B347, 6 (1995).

[16] Geiger, K. and Ellis, J., Nucl. Phys. A590, 609c (1995)

[17] Ghosh, S. K., Talk at 1997 Yukawa International Seminar on Non-perturbative QCD, Kyoto, Japan, December 1997.

[18] Chandrasekhar, S., Rev. Mod. Phys. 15, 1 (1943).

[19] Florkowski, W. and Friman, B. L., Acta Phys. Pol. 25, 49 (1994).

[20] Altherr, T. and Seibert, D., Phys. Rev. C49, 1684 (1994).

[21] Traxler, C. T. and Thoma, M. H., Phys. Rev. C53, 1348 (1996).

[22] Geiger, K.,Phys. Rev. D46, 4965 (1992).

[23] Plümer, M., Raha, S. and Weiner, R. M., Phys. Lett. B139, 198 (1984); Nucl. Phys. A418, 549c (1984).

[24] Jaminon, M., This volume. 